\title{
Pericardial tamponade caused by Pasteurella multocida infection after a cat bite
}

\author{
A K Al-Allaf, T C Harvey, A R Cunnington
}

\begin{abstract}
An unusual case of meningitis and pericardial tamponade caused by Pasteurella multocida after a cat bite is reported. The patient was successfully treated by antibiotics and pericardiocentesis and made an uneventful recovery from a life threatening condition. This case illustrates the potential dangers that can arise from a seemingly trivial and commonplace injury.

(Postgrad Med f 2001;77:199-200)
\end{abstract}

Keywords: pasteurellosis; pericardial tamponade; cat bite

\section{Case report}

A 62 year old women was admitted with a 48 hour story of headache, neck stiffness, central pleuritic chest pain relieved by sitting forward and made worse by lying flat, fever, rigors, and drowsiness. Four days earlier she had been badly mauled by a stray cat and had sustained bites and lacerations to her right wrist.

On examination she was unwell, drowsy, dehydrated, and had a fever of $39.8^{\circ} \mathrm{C}$. She had an obviously inflamed bite and scratch marks on her wrist with local cellulitis. Her blood pressure was $106 / 59 \mathrm{~mm} \mathrm{Hg}$, pulse $84 /$ minute, regular and oxygen saturation was $96 \%$. Heart sounds at this stage appeared normal with no murmurs or rub and chest examination was clear. There were no focal neurological signs but she was drowsy and confused and the provisional diagnosis was of meningoencephalitis and pericarditis due to septicaemia or viraemia as a result of the injury.

\section{Investigations}

Her haemoglobin concentration was $132 \mathrm{~g} / 1$ and white cell count $18.6 \times 10^{9} / 1 \quad(90 \%$ neutrophils). Sodium concentration was 122 $\mathrm{mmol} / \mathrm{l}$, potassium $3.2 \mathrm{mmol} / \mathrm{l}$, urea 10.1 $\mathrm{mmol} / \mathrm{l}$, creatinine $65 \mu \mathrm{mol} / 1$, erythrocyte sedi-

Submitted 4 February 2000 Accepted 22 June 2000
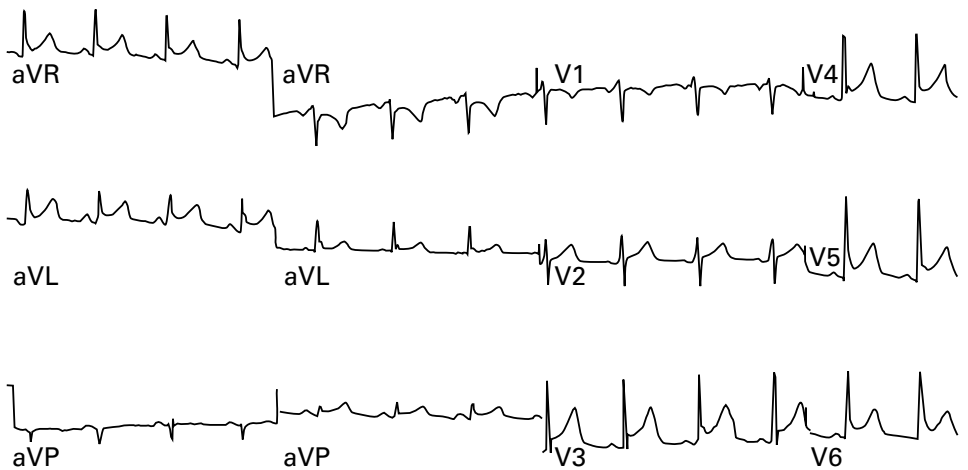

Figure 1 A 12 lead electrocardiogram on admission shows ST elevation with upward concavity suggestive of acute pericarditis.

\section{Learning points}

- Pasteurellosis is a common infection after animal bite.

- Infection may range from trivial local inflammation to life threatening septicaemia with multiorgan involvement.

- Pericardial tamponade should be considered in treating critically ill patient with this infection.

mentation rate $70 \mathrm{~mm} /$ hour, $\mathrm{C}$ reactive protein $316 \mathrm{mg} / \mathrm{l}$, immunoglobulins and liver function tests were normal, and an autoimmune screen was negative. Chest radiography was normal. Electrocardiography showed diffuse ST segment elevation with upward concavity (fig 1); computed tomography of the skull was normal. Blood cultures grew Gram negative coccobacilli (later identified as Pasteurella multocida, sensitive to a variety of antibiotics including amoxicillin and cefotaxime). Lumbar puncture 72 hours after admission showed white cell count of $50 \times 10^{6} / 1$ (100\% neutrophils), a glucose of $4.2 \mathrm{mmol} / \mathrm{l}$, and protein of $0.65 \mathrm{~g} / 1$. Culture was sterile (on antibiotics).

The patient was started on intravenous antibiotics (flucloxacillin and cefotaxime), rehydrated, and closely monitored. Over the first 72 hours her temperature settled but she developed atrial fibrillation and became progressively more confused and comatose with increasing hypoxia, hypotension, and diminished urine output, requiring ventilation and inotropic support. Swan-Ganz catheterisation revealed a low cardiac output with high filling pressures and increased peripheral vascular resistance. Echocardiography revealed a large pericardial effusion with early diastolic collapse of the right atrium suggestive of tamponade (fig 2).

Altogether $1150 \mathrm{ml}$ of turbid fluid was aspirated by pericardiocentesis and a pericardial drain inserted. There was immediate haemodynamic improvement with a rise in systolic pressure from 85 to $220 \mathrm{~mm} \mathrm{Hg}$ and the atrial fibrillation reverted to sinus rhythm during the procedure.

Her subsequent progress was uneventful. She regained consciousness and went on to make a good recovery over the next two weeks.

\section{Discussion}

Cardiac tamponade is a relatively uncommon sequela of a bacteraemia and may present with a wide spectrum of clinical features ranging from an asymptomatic elevation of intrapericardial pressure recognised during objective evaluation, to extreme haemodynamic com- 

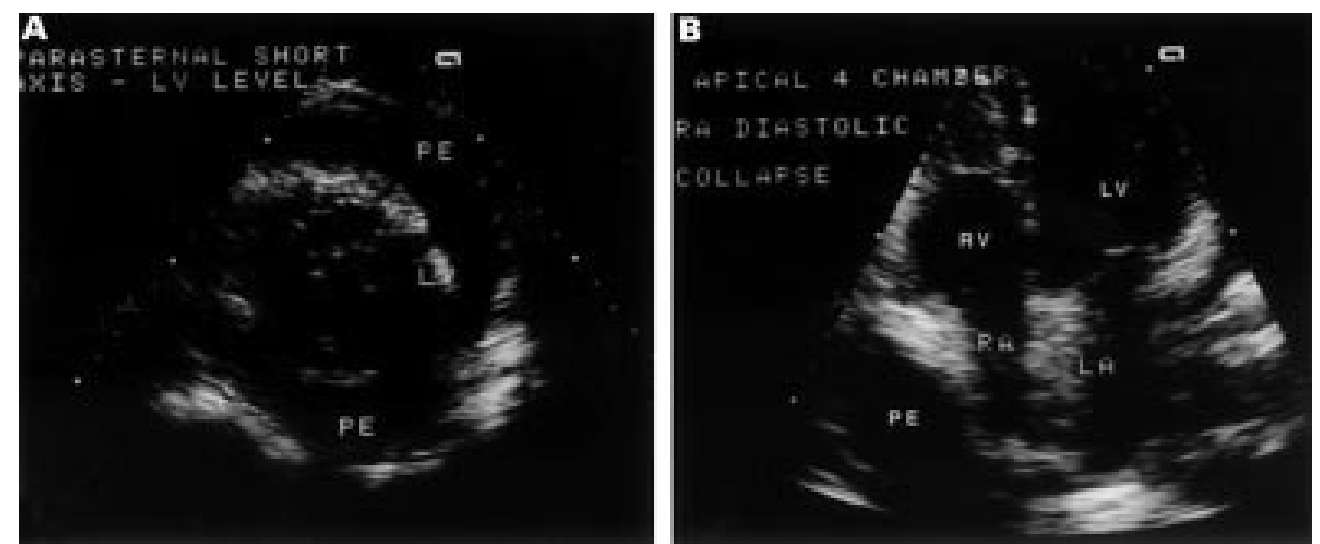

Figure 2 (A) Parasternal short axis view of the transthoracic echocardiography demonstrating the presence of a large echo free area due to the pericardial effusion (PE) surrounding the left ventricle (LV). (B) Apical four chamber view of the transthoracic echocardiography in diastole. The right atrium $(R A)$ is compressed by the pericardial effusion (PE) impending venous inflow to the heart ( $L A=$ left atrium, $L V=$ left ventricle, $R V=$ right ventricle).

promise in the form of severe hypotension or electromechanical dissociation. ${ }^{1}$ If the fluid in the pericardium is not quickly removed, the patient will die from cardiogenic shock. ${ }^{2}$ The diagnosis is made from the clinical history, physical findings, and radiological and electrocardiographic suspicion and is easily confirmed by echocardiography.

Pasteurellosis, a zoonosis, is caused by a Gram negative coccobacilli found in the nasopharynx and gastrointestinal tract of many animals. About $50 \%-90 \%$ of domestic cats and dogs carry pasteurella species in their saliva and nasal tract and the organism is frequently found in injuries from cat scratches $(75 \%)$ and dog bites $(50 \%)$. $^{3}$

Pasteurella multocida may cause local wound infections in man as a result of contact with animals and serious systemic infection and septicaemia has been reported including endo- carditis, pericarditis, ${ }^{4}$ polyarthropathy, and death. ${ }^{5}$ This case is believed to be the first reported instance of pasteurella pericardial tamponade.

The interesting aspect about this illness is that it was caused by a cat bite. This emphasises the importance of considering this unusual infection in all animal inflicted injuries.

1 Ameli S, Shah PK. Cardiac tamponade: pathophsiology, diagnosis, and management. Cardiol Clin 1991;9:665-74.

2 Minutiello L. Cardiac tamponade: emergency pericardiocentesis using a common set for central venous catheterisation. Minerva Cardioangiol 1996;44:209-20.

3 Talan DA, Citron DM, Abrahamian FM, et al. Bacteriologic analysis of infected $\operatorname{dog}$ and cat bites. $N$ Engl $f$ Med 1999;340:85-92.

4 Sorbello AF, O'Donnell J, Kaiser-Smith J, et al. Bacterial endocarditis due to Pasteurella dagmatis. Clin Infect Dis 1994;18:336-8.

5 Jenkins R. Pensioner killed by cat she rescued. The Times 2 October 1999. 